International Journal of Applied Mathematical Research, $6(4)(2017) 121-124$
International Journal of Applied Mathematical Research
Website: www.sciencepubco.com/index.php/IJAMR
doi: $10.14419 /$ ijamr.v6i4.7914
Research paper

\title{
The pricing of spread option using simulation
}

\author{
Hamid Reza Erfanian *, Seyed Jaliledin Ghaznavi Bidgoli, Parvin Shakibaei \\ Department of Financial Mathematics, University of Science and Culture, Tehran, Iran \\ *Corresponding author E-mail: erfanian@usc.ac.ir
}

\begin{abstract}
Monte Carlo simulation is one of the most common and popular method of options pricing. The advantages of this method are being easy to use, suitable for all kinds of standard and exotic options and also are suitable for higher dimensional problems. But on the other hand Monte Carlo variance convergence rate is $\mathrm{O}\left(\mathrm{n}^{-1 / 2}\right)$ which due to that it will have relatively slow convergence rate to answer the problems, as to achieve $\varepsilon$ accuracy when it has been d-dimensions, complexity is $\mathrm{O}\left(\mathrm{d}^{-3}\right)$. For this purpose, several methods are provided in quasi Monte Carlo simulation to increase variance convergence rate as variance reduction techniques, so far. One of the latest presented methods is multilevel Monte Carlo that is introduced by Giles in 2008. This method not only reduces the complexity of computing amount $\mathrm{O}\left(\varepsilon^{-3} \log \left(\varepsilon^{2}\right)\right)$ in use of Euler discretization scheme and the amount $\mathrm{O}\left(\varepsilon^{-2}\right)$ in use of Milstein discretization scheme, but also has the ability to combine with other variance reduction techniques. In this paper, using Multilevel Monte Carlo method by taking Milstein discretization scheme, pricing spread option and compared complexity of computing with standard Monte Carlo method. The results of Multilevel Monte Carlo method in pricing spread options are better than standard Monte Carlo simulation.
\end{abstract}

Keywords: Monte Carlo Simulation; Multilevel Monte Carlo; Spread Options Pricing.

\section{Introduction}

Currently, due to the vast development of financial markets and investor's demand, application of financial derivatives as new financial method in financial transactions, has been inevitable. The most important examples of financial derivatives are option contracts. The Contracts, which are currently highly traded, by reason of immense variety, low price regarding to underlying asset and also in order to meet investor's basic need (requirements) in financial markets. The variety of option contracts and their dependence on the assumed models of the underlying asset has challenged the pricing of these contracts.

In general, pricing methods for option contracts are classified into two categories: analytical and numerical methods. Since the first category is used only in certain and limited circumstances, numerical methods are more common and more useful. One of the numerical methods in pricing option contracts is Monte Carlo simulation method. The advantages of this method are the ease of use, being suitable for higher dimensional problems and suitability for all kinds of standard and exotic options.

On the other hand, Monte Carlo method has a relatively slow rate of convergence rate solving a problem, such that in order to achieve accuracy $\varepsilon$, for a problem of dimension-d, the complexity is $\mathrm{O}\left(\mathrm{d}^{-3}\right)$.[1] Also Monte Carlo variance convergence rate, known as Canonical Monte Carlo convergence rate, is $\mathrm{O}\left(\mathrm{n}^{-1 / 2}\right)$. To increase the efficiency of Monte Carlo methods, variance reduction techniques have been proposed. These methods fall into two main groups: Control variables and changes in the random input[2]. One of the novel approaches to reduce the variance, which is based on the control variables techniques, is Multilevel Monte Carlo method (MLMC), that has been introduced by Giles[3]. In a Multilevel Monte Carlo method, the computational complexity reduces from $O\left(\varepsilon^{-3}\right)$ to $O\left(\varepsilon^{-3} \log \left(\varepsilon^{2}\right)\right)$, by applying different time steps, when the Euler discretization scheme method has been used for the simulation of the processes underlying asset. Multilevel methods are easy to implement and have the ability to combine with other variance reduction techniques [4]. In this paper, we aim to reduce the complexity of calculations and thus provide a more efficient method, by applying multilevel Monte Carlo method and considering the Milstein discretization scheme method in pricing Spread options.

The structure of this paper is as follows. In the second part of this article, the empirical studies and research background of a multilevel Monte Carlo method will be reviewed. Third part discusses the required concepts and definitions. In the fourth section we present multi-level Monte Carlo method. Lastly in the fifth section, the results of Monte Carlo simulation in pricing Spread options by Milstein discretization scheme method, will be mentioned and compared to the standard Monte Carlo method, in terms of (with respect to the) computational complexity.

\section{Theoretical backgrounds and an overview of the research history}

The idea of reducing the cost of computation by multi-level Monte Carlo method was first introduced by Giles in 2008. He demonstrated, that in order to achieve an accuracy equivalent to $O(\varepsilon)$, for Lipschitz payoff and Euler discretization scheme method, computational cost decreases from $\mathrm{O}\left(\varepsilon^{-3}\right)$ to $\mathrm{O}\left(\varepsilon^{-3} \log \left(\varepsilon^{2}\right)\right)$. Also has priced European option, Asian option, Lookback option and Digital option in the numerical results with respect to the geometric Brownian motion as a model of the underlying asset, and to vast more this method has calculated European option price when the underlying asset follows the Heston stochastic volatility model in a brief example[4]. 
In the same year, Giles had improved multilevel Monte Carlo method by changing (altering) the discrete stochastic differential equation method and using Milstein discretization scheme method and Showed using (applying) Milstein discretization scheme method in multilevel Mont Carlo simulation, decreases the computational cost to $\mathrm{O}\left(\varepsilon^{-2}\right)$. In (that/ the mentioned) paper he represents numerical results for Asian option, Lookback option, Barrier option and Digital option[5].

In 2009 Giles and Waterhouse offered a multilevel quasi-Monte Carlo method by combining a variance reduction method and multi-level Monte Carlo method. They combined multi-level Monte Carlo method with multilevel quasi-Monte Carlo Rank-1 lattice rule method and showed the resulting method have lower computational cost in pricing of Asian option, Lookback option, Barrier option and Digital option [6]. In the same year, Giles et al. expanded a multilevel Monte Carlo method for Non-globally Lipschitz payoff [7]. In 2009, Giles uses multilevel Monte Carlo method for Basket options. He demonstrates in this paper that multi-level Monte Carlo method is easily applicable for Basket options and also shows that numerical results for Asian basket, Prevent basket, Digital basket and Lookback basket has computational cost equivalent to $O\left(\varepsilon^{-2}\right)$ [8].

In 2011, Primozic uses multi-level Monte Carlo method to estimate the expected value for First passage times in Stochastic process [9]. In the following year, Huth proposed an application of multi-level Monte Carlo method for Asian option and American option by combining a variance reduction method and multi-level Monte Carlo method. He introduced a more efficient Multi Level quasi-Monte Carlo method [3]. Recently in 2014, Ferreiro-Castilla et al. presented an article on multilevel Monte Carlo simulation for Levy processes based on Wiener-Hopf factorization [10].

\section{Definitions and backgrounds}

In this paper, the payoff function of spread option has been considered as the subtraction of $S_{1}$ from $S_{2}$ as follows ( $\mathrm{K}$ is the strike price ) [11]:

$\left(S_{1}(t)-S_{2}(t)-K\right)^{+}$

In addition it assumes that the base asset follows Samuelson stochastic differential equation as below[12]:

$d S_{1}=S_{1}\left(r-\delta_{1}\right) d t+\sigma_{1} S_{1} d B$

$d S_{2}=S_{2}\left(r-\delta_{2}\right) d t+\sigma_{2} S_{2} d B_{2}$

When the interest rate $\mathrm{r}, \delta_{i}$ the steady dividends rate, $\sigma_{i}$ the standard deviation per share, $B_{1}$ and $B_{2}$ are two Brownian motion with a correlation coefficient of $\rho$.

Based on Martingale's pricing theory, the spread option price of $p$ at time $t$ is obtained by computing the expected value of the absolute risk's of Q, as follows:

$p\left(S_{1}(t), S_{2}(t), K, T\right)=e^{-r(T-t)} \mathrm{E}_{\mathrm{Q}}\left[\left(S_{1}(t)-S_{2}(t)-K\right)^{+} \mid \mathrm{F}_{\mathrm{t}}\right]$

In the above equation $\mathrm{T}$ is maturity time and $F_{t}$ is filter until time $\mathrm{t}[2]$.

The above equation is used for pricing option contract in Monte Carlo simulation. But since the simulation of basic assets requires the existence of a strong solution for the given differential equations- which is often not feasible for most of mentioned processesdiscrete methods (procedures) of stochastic differential equation are used for the simulation of Monte Carlo method. In the following, two methods of Euler discretization scheme and Milstein discretization scheme are provided briefly [13].

\subsection{Euler discretization scheme method}

Assume $S=(S(t))_{\mathrm{t}[0, \mathrm{~T}]}$ is an Ito process and in the following stochastic differential equation over $0 \leq t \leq T$, with the initial condition $S=S_{0}$, is valid:

$d S(t)=a(S(t), t) d t+b(S(t), t) d B(t)$

For the given discretized time $0=t_{0} \leq \ldots \leq t_{m}=T, \Delta t_{i}=t_{i}-t_{i-1}$ and $\Delta B_{i}=B_{t_{i}}-B_{t_{i-1}}$, Euler estimation is a random continuous-time process that is achieved by the below recursive (Iterative) procedure:

$\hat{S_{0}}=S_{0}$

$\hat{S_{i+1}} \approx \hat{S_{i}}+a\left(\hat{S}_{i}, t_{i}\right) \Delta t_{i}+b\left(\hat{S_{i}}, t_{i}\right) \Delta B$

It should be noted that the Euler discretization scheme method has a strong convergence rate of $O\left(h^{1 / 2}\right)$

\subsection{Milstein discretization scheme method}

Considering the stochastic differential equation (5), Milstein discretization scheme method is computed as follows:

$\hat{S_{0}}=S_{0}$

$\hat{S}_{i+1} \approx \hat{S}_{i}+a\left(\hat{S}_{i}, t_{i}\right) \Delta t_{i}+b\left(\hat{S}_{i}, t_{i}\right) \Delta B_{i}+\frac{1}{2} b \frac{\partial b}{\partial S}\left\{\frac{1}{2}\left(\Delta B_{i}\right)^{2}-\frac{1}{2} \Delta t_{i}\right\}$

It should be noted that the Milstein discretization scheme method has a strong convergence rate of $\mathrm{O}(\mathrm{h})$.

\section{Multi-level Monte Carlo method}

The basic idea of multilevel Monte Carlo method is to change the time steps in different levels and ultimately create a new and more efficient (adequate) estimator.

We consider Monte Carlo simulations at different time steps $h_{l}=M^{-l} T$ for $l=0,1, \ldots, L$ (M is filter operation), when $\mathrm{P}$ indicates the payoff function , $\hat{P}_{l}$ and $\hat{S}_{\nu^{\prime}}$ are Indicative estimates for $\mathrm{p}$ and $\mathrm{S}(\mathrm{T})$, by using numerical discretization with the step size of $h_{l}$.

Next (Now), due to the linearity of the expected value, expected value of PL could be calculated as follows:

$E\left[P_{L}\right]=E\left[P_{0}\right]+\sum_{l=1}^{L} E\left[P_{l}-P_{l-1}\right]$

Multi-level Monte Carlo method aims to minimize the computational cost by estimating each expected value of the right side of equality (8). So the estimators of each level are defined as follows:

$\hat{\mathrm{Y}}_{1}=\left\{\begin{array}{l}\frac{1}{N_{0}} \sum_{l=1}^{N_{0}} P_{0}^{(i)}, l=0 \\ \frac{1}{N_{l}} \sum_{l=1}^{N_{l}} P_{l}^{(i)}-P_{l-1}{ }^{(i)}, l=1, \ldots, L\end{array}\right.$

Where $N_{l}$ is the number of paths simulated in the lth level and the multi-level Monte Carlo estimator is equal to:

$\hat{Y_{L}}=\hat{Y_{0}}+\sum_{l=1}^{L} \hat{Y_{l}}$

There are two key points in the multi-level Monte Carlo estimator. First, in the estimators of $\hat{Y}$, for each level $l=0,1, \ldots, L$, (the) num- 
ber of simulated paths for $N_{1}$ are changing and second, for $\hat{Y}$, estimators with $l \geq 1$, the procedure of simulating is the same as Brownian growth but with two different time steps.

\subsection{Complexity theorem}

The complexity theorem is used by Giles under certain circumstances, to determine the results of computational complexity of multi-level Monte Carlo method [4, 5]. He also showed that it is possible to reduce general complexity of Monte Carlo simulation from $O\left(\varepsilon^{-3}\right)$ to $O\left(\varepsilon^{-2}\right)$ under these conditions. This theorem is described generally and it is valid for a variety of underlying asset processes and payoff functions, which are not necessarily Lipchitz.

\subsubsection{Theorem}

Suppose $\mathrm{P}$ indicates a function from solving the stochastic differential equation (5). Also Suppose $\hat{P}_{l}$ represents the estimation of numerical discretization with time steps $h_{l}=M^{-l} T$. If independent estimators $\left(\hat{Y_{l}}\right)$, consists of $N_{l}$ as Mont Carlo samples and $C_{l}$ as computational cost, furthermore positive constants $c_{1}, c_{2}, c_{3}, \beta, \alpha \geq \frac{1}{2}$ exist and if:

i) $\quad E\left[\hat{P}_{l}-P\right] \leq c_{1} h_{l}^{\alpha}$

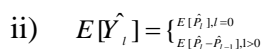

iii) $V\left[\hat{Y_{l}}\right]<\mathrm{c}_{2} \mathrm{~N}_{1}^{-1} \mathrm{~h}_{1}^{P}$

iv) $C_{l} \leq c_{3} N_{l} h_{l}^{-1}$

Then there exists a positive constant, $c_{4}$, when for each $\varepsilon<e^{-1}$ values of $\mathrm{L}$ and $N$, for multi-level estimator of

$\hat{Y}=\sum_{l=0}^{L} \hat{Y_{l}}$

Exist and this estimator has a bounded mean squared error,

$\mathrm{MSE} \equiv \mathbb{E}\left[(\widehat{\mathrm{Y}}-\mathbb{E}[\mathrm{P}])^{2}\right]<\varepsilon^{2}$

And the computational cost $\mathrm{C}$ as follows:

$C \leq\left\{\begin{array}{c}\mathrm{c}_{4} \varepsilon^{-2} \beta>1 \\ \mathrm{c}_{4} \varepsilon^{-2}(\log \varepsilon)^{2} \beta=1 \\ \mathrm{c}_{4} \varepsilon^{-2-(1-\beta) / \alpha} 0<\beta<1 .\end{array}\right.$

This theorem refers to the importance of the value of $\beta$ parameter, which is the definition (concept) of convergence (of) variance $V_{1}$, when $1 \rightarrow \infty$. In Euler discretization scheme method and Lipschitz payoff function $\beta$ will be $1(\beta=1)$. Therefore, the computational cost of Monte Carlo estimator is equal to $O\left(\varepsilon^{-2}(\log \varepsilon)^{2}\right)$. That is, if we use Milstein discretization scheme method (instead of Euler discretization scheme method, to approximate stochastic differential equation, it's strong convergence of $\mathrm{O}(\mathrm{h})$ for Lipschitz payoff functions, results an accuracy level of $O\left(h_{l}^{2}\right)$ for variance estimator of each level. In other words, with Milstein discretization scheme method, $\beta=2$ and thus the cost of computing for multilevel Monte Carlo estimator is $O\left(\varepsilon^{-2}\right)$ [4]. It has to be mentioned that the above results by Giles, are also provided for non-public Lipschitz payoff functions [7].

\subsection{Numerical algorithm}

Giles has provided a numerical algorithm to run Multi-level Monte Carlo simulation as follows:
1) Initialize $L=0$

2) Estimate $V_{L}$ using $N_{L}=2 \times 10^{5}$ prototype sample

3) Define Optimized $N_{l}, l=0, \ldots, L$ by using the following equation:

$N_{l}=\left\lceil 2 \varepsilon^{-2} \sqrt{V_{L} h_{l}}\left(\sum_{l=0}^{L} \sqrt{V_{l} / h_{l}}\right)\right\rceil$

4) Evaluate the rest of samples in each layer to check if a new $N_{l}$ is required or not.

5) If $L \geq 2$, do the convergence test by the following equation $\max \left\{M^{-1}\left|\hat{Y}_{L-1}\right|,\left|\hat{Y}_{L}\right|\right\}<\frac{1}{2}(M-1) \varepsilon$ (11)

6) If $L<2$ or non-convergent, then $\mathrm{L} \rightarrow \mathrm{L}+1$ and return to number 2 .

This algorithm estimates the multilevel variance estimator, less than $\varepsilon^{2} / 2$. Additionally with a specified error $\varepsilon$, this algorithm provides, a mean square error (MSE) less than $\varepsilon^{2}$ [4].

\section{Simulation results}

In this section, we represented the results of multi-level Monte Carlo simulations, using Milstein discretization scheme method for spread options. The results has been obtained by the given values of $S_{1}=90, S_{2}=80, r=0.05, \sigma_{1}=0.2, \sigma_{2}=0.2, \rho=0.5$, $K=5$ and $T=1$. Also $\mathrm{M}$ is considered $\mathrm{M}=2$, because the minimum possible value for filter element, based on equation (11), increases the speed of convergence in the numerical algorithm [4].

Table 1: Compares the Computational Cost of MLMC to MC for Spread Options

\begin{tabular}{lllll}
\hline \multicolumn{1}{c}{$\varepsilon$} & $\widehat{\mathrm{P}}_{\text {Spread }}$ & MLMC_cost & MC_cost & $\frac{\text { MC_cost }}{\text { MLMC_cost }}$ \\
\hline 0.001 & 9.0994 & 296900000 & 2635000000 & 8.87 \\
0.005 & 9.1067 & 12390000 & 49180000 & 3.97 \\
0.01 & 9.0860 & 2865000 & 12300000 & 4.29 \\
0.05 & 9.1042 & 118600 & 491800 & 4.15 \\
0.1 & 8.9766 & 28600 & 123000 & 4.30 \\
\hline
\end{tabular}

In Table 1, the estimated price of spread options and a comparison of computational cost is provided for two methods, multi-level Monte Carlo (MLMC) and Monte Carlo (MC) with different values of error. Evidently, MLMC method (with) error of $\varepsilon=0.001$ is approximately 8 times more efficient than MC method.

Other results of MLMC simulation is shown in Figure 1. The above graph in left, demonstrates the behavior of the estimator's variance, in both standard Mont Carlo $\left(\hat{P}_{l}\right)$ and multi-level Monte Carlo $\left(\hat{P}_{l}-\hat{P}_{l-1}\right)$. The drawn quantity of logarithm to the base 2 for two estimators has been illustrated next to each level. In order to graph, the slope of the $\hat{P}_{l}-\hat{P}_{l-1}$ estimator is approximately 2, which imply that $V_{l}=V\left[\hat{P}_{l}-\hat{P}_{l-1}\right]=\mathrm{O}\left(\mathrm{h}_{1}^{2}\right)$. When $L=2$; which in this case, the simulation time step is only 4 , the variance of estimator MLMC is at least 2000 times smaller than the variance of estimator MC. Top right graph shows that $E\left[\hat{P}_{l}-\hat{P}_{l-1}\right]$ has a rate of $O\left(\mathrm{~h}_{1}\right)$ According to equation (11), this value is used to determine the required number of levels, to reduce the bias to an acceptable level. Two bottom graphs in figure 1, show the result of 5 simulations of MLMC with different values of error. The bottom left graph, represents the number of optimal routes at different levels to achieve the desired error. For example, when $\varepsilon=0.005$, simulation would be performed up to two level and with the specified number of optimal routes in Table 2. The Bottom graph in the right also shows the computational cost of the two methods, MLMC and MC. 

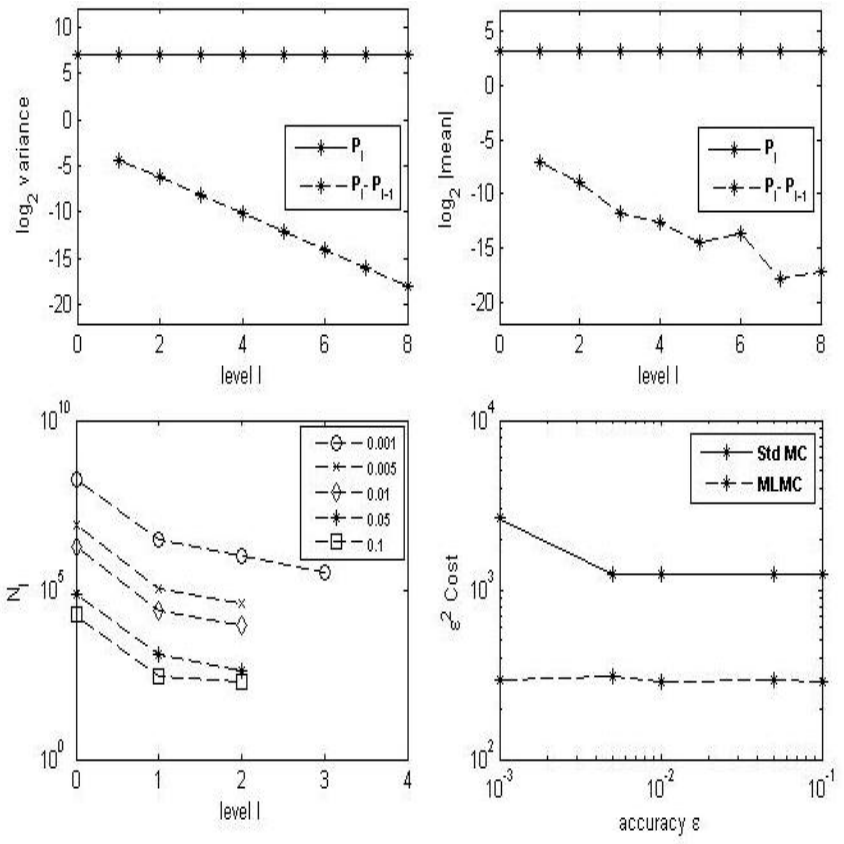

Fig. 1: The Results of A MLMC Simulation for Spread Options.

Table 2: Simulation of optimal routes MLMC for spread options

\begin{tabular}{rllll}
\hline \multicolumn{1}{c}{$\varepsilon$} & \multicolumn{1}{c}{$N_{0}$} & \multicolumn{1}{c}{$N_{1}$} & \multicolumn{1}{c}{$N_{2}$} & \multicolumn{1}{c}{$N_{3}$} \\
\hline 0.001 & 185627057 & 2903337 & 938794 & 340567 \\
0.005 & 7911664 & 100191 & 37287 & - \\
0.01 & 1824542 & 24796 & 9043 & - \\
0.05 & 74984 & 1221 & 407 & - \\
0.1 & 17696 & 285 & 200 & - \\
\hline
\end{tabular}

\section{Conclusion}

As we have seen, convergence rate of multi-level variance estimator rate has a degree of $O\left(\mathrm{~h}_{1}{ }^{2}\right)$, while the conventional convergence rate of Monte Carlo method is constant and equal to $O\left(n^{-1 / 2}\right)$.Also the results of cost computation, emphasized that multilevel Monte Carlo method in spread options is more efficient and appropriate than standard Monte Carlo method.

\section{References}

[1] Wilmott, P., Frequently asked questions in quantitative finance 2010: John Wiley \& Sons.

[2] Glasserman, P., Monte Carlo methods in financial engineering. Vol. 53. 2003: Springer Science \& Business Media https://doi.org/10.1007/978-0-387-21617-1.

[3] Huth, D., Application of Multilevel Monte Carlo simulation to Barrier, Asian and American options, in Mathematics2012, Technical University of Munich.

[4] Giles, M.B., Multilevel monte carlo path simulation. Operations Research, 2008 a. 56(3): p. 607-617. https://doi.org/10.1287/opre.1070.0496.

[5] Giles, M., Improved multilevel Monte Carlo convergence using the Milstein scheme, in Monte Carlo and quasi-Monte Carlo methods 2006. 2008 b, Springer. p. 343-358. https://doi.org/10.1007/978-3540-74496-2 20.

[6] Giles, M.B. and B.J. Waterhouse, Multilevel quasi-Monte Carlo path simulation. Advanced Financial Modelling, Radon Series on Computational and Applied Mathematics, 2009: p. 165-181.

[7] Giles, M.B., D.J. Higham, and X. Mao, Analysing multi-level Monte Carlo for options with non-globally Lipschitz payoff. Finance and Stochastics, 2009. 13(3): p. 403-413 https://doi.org/10.1007/s00780-009-0092-1.

[8] Giles, M.B. Multilevel Monte Carlo for basket options. in Simulation Conference (WSC), Proceedings of the 2009 Winter. 2009. IEEE.

[9] Primozic, T., Estimating expected first passage times using multilevel Monte Carlo algorithm, in Mathematical and Computational Finance2011, University of Oxford.
[10] Ferreiro-Castilla, A., et al., Multilevel Monte Carlo simulation for Lévy processes based on the Wiener-Hopf factorisation. Stochastic Processes and their Applications, 2014. 124(2): p. 985-1010. https://doi.org/10.1016/j.spa.2013.09.015.

[11] Zhang, P.G., Exotic Options: A Guide to Second Generation Options. 1998: World Scientific. https://doi.org/10.1142/3800.

[12] Andersson, M., Valuation of spread options using the fast Fourier transform under stochastic volatility and jump diffusion models. 2015.

[13] Heinrich, S., Multilevel monte carlo methods, in Large-scale scientific computing. 2001, Springer. p. 58-67. https://doi.org/10.1007/3540-45346-6_5. 